\title{
BMJ Open Relationship between maternal healthcare utilisation and empowerment among women in Bangladesh: evidence from a nationally representative cross-sectional study
}

\author{
Asibul Islam Anik (D) , ${ }^{1,2}$ Bishwajit Ghose (D) , ${ }^{3,4}$ Md. Mosfequr Rahman (1) ${ }^{2}$
}

To cite: Anik Al, Ghose B, Rahman MM. Relationship between maternal healthcare utilisation and empowerment among women in Bangladesh: evidence from a nationally representative

cross-sectional study. BMJ Open 2021;11:e049167. doi:10.1136/ bmjopen-2021-049167

- Prepublication history and additional supplemental material for this paper are available online. To view these files, please visit the journal online (http://dx.doi.org/10.1136/ bmjopen-2021-049167)

Received 18 January 2021 Accepted 02 August 2021

\section{Check for updates}

\section{Author(s) (or their} employer(s)) 2021. Re-use permitted under CC BY-NC. No commercial re-use. See rights and permissions. Published by BMJ.

${ }^{1}$ Department of Public Health and Informatics, Bangabandhu Sheikh Mujib Medical University, Dhaka, Bangladesh

${ }^{2}$ Population Science and Human Resource Development, University of Rajshahi, Rajshahi, Bangladesh

${ }^{3}$ Institute of Nutrition and Food Science, University of Dhaka,

Dhaka, Bangladesh

${ }^{4}$ School of Nursing, University of Ottawa, Ottawa, Ontario, Canada

Correspondence to Dr Md. Mosfequr Rahman; mosfeque@ru.ac.bd

\section{ABSTRACT}

Objective To examine the relationship between women's empowerment and maternal healthcare utilisation in Bangladesh.

Design This cross-sectional study uses data from the most recent nationally representative Bangladesh Demographic and Health Survey, 2017-2018.

Setting Bangladesh.

Participants Married women aged 15-49 years who had a live birth within the 3 years preceding the survey $(n=4767)$.

Primary and secondary outcome measures Women's empowerment was measured using the recently developed and validated survey-based Women's emPowERment (SWPER) index. The index includes three domains: social independence, decision-making and attitude to violence. Outcomes included utilisation of at least one antenatal care from skilled providers (ANC1), at least four antenatal care visits ( $\geq 4 \mathrm{ANC}$ ), delivery assisted by a skilled birth attendant (SBA) and a postnatal visit within 2 days of delivery (PNC). Logistic regression analyses were used to assess the identified relationships.

Results Among participants, 83\% received ANC1, 46.3\% received $\geq 4$ ANC, $51.9 \%$ reported SBA and $50.9 \%$ sought PNC. Women with high levels of social empowerment relative to those with low levels were more likely to use ANC1 (adjusted OR (AOR) 1.85; 95\% Cl 1.40 to 2.45), $\geq 4$ ANC (AOR 1.55; 95\% Cl 1.27 to 1.90), SBA (AOR 2.12; 95\% Cl 1.71 to 2.62 ) and PNC (AOR 1.95; 95\% Cl 1.56 to 2.44). Compared with women with low levels of decision-making empowerment, women with high levels were more likely to use SBA (AOR 1.49; $95 \% \mathrm{Cl} 1.21$ to 1.83 ) and PNC (AOR $1.47 ; 95 \% \mathrm{Cl} 1.19$ to 1.81 ). Additionally, significant inequality was observed among women moving from low to high empowerment in all domains of the empowerment index. Conclusions Higher empowerment levels were positively associated with maternal healthcare utilisation in Bangladesh. Our findings suggest the need to address women's empowerment in policies aiming to expand health service utilisation.

\section{INTRODUCTION}

The burden of maternal mortality in developing countries remains unacceptably

\section{Strengths and limitations of this study}

- This study used a large sample size encompassing both rural and urban areas and the most recent nationally representative study population.

We used the newly developed SWPER (survey-based Women's emPowERment) index, which captures three dimensions of women's empowerment.

- The data used in this study are cross-sectional, and therefore, it is not possible to establish a causal relationship between different dimensions of women's empowerment and the utilisation of maternal healthcare services.

high despite significant progress in recent decades. Globally, approximately 810 women died every day in 2017 due to pregnancy and childbirth-related causes, and the majority of these deaths (94\%) occurred in developing countries. ${ }^{1}$ Although the maternal mortality ratio (MMR) in Bangladesh substantially declined from 574 to 176 deaths per 100000 live births between 1990 and 2015, a 69\% reduction, ${ }^{2}$ the country is still far from achieving the global target set by the United Nations Sustainable Development Goal (SDG) 3, which specifies a reduction to 70 maternal deaths per 100000 live births by 2030. Many maternal deaths in Bangladesh as well as globally could be prevented with adequate and proper utilisation of maternal healthcare services (MHS). ${ }^{1}$

Despite this impressive decline in the MMR in Bangladesh, women's use of basic MHS from skilled professionals remains low. ${ }^{3}$ The most recent Bangladesh Demographic and Health Survey (BDHS) 2017-2018 reported that $47 \%$ of women attended four or more antenatal care (ANC) visits during their last pregnancy as recommended by the WHO, $53 \%$ of deliveries were attended by medically 
trained providers and $52 \%$ of mothers obtained a postnatal check-up within 2 days of delivery. ${ }^{4}$ WHO advocates for increased utilisation of health facilities during pregnancy and delivery, which is of paramount importance in reducing maternal mortality. ${ }^{5}$ Extensive research from developing countries has attempted to identify various sociodemographic, geographic and cultural correlates associated with utilisation of MHS from skilled professionals. ${ }^{3}{ }^{6-9}$ A handful of studies from developing countries, ${ }^{10-14}$ including Bangladesh, ${ }^{15-20}$ has documented the relationship between women's empowerment and maternal healthcare utilisation. However, these studies were mainly focused on women's involvement in household decision-making, ${ }^{10-14}$ 16-18 20 freedom of movement $^{111718}$ or attitude towards violence, ${ }^{12} 131819$ which provide a narrow scope of women's empowerment for understanding this relationship.

Therefore, this study aims to examine the relationship between women's empowerment and maternal healthcare utilisation in Bangladesh using the newly developed survey-based Women's emPowERment (SWPER) index to guide this empirical investigation. ${ }^{212}$ Due to its abstract and complex nature, women's empowerment is difficult to measure. ${ }^{23}$ Empowerment is multidimensional and can be expressed at multiple levels. However, which dimensions or levels are most important is a point of argument. Based on the empowerment module of the Demographic and Health Survey (DHS), several indices have been proposed in developing countries that include questions about women's participation in household decisionmaking; opinions on wife beating; control over resources; employment and type of earnings; and personal ownership of land or a house. ${ }^{10-13161724}$ These indices are subjectively weighted and are designed for specific countries or small groups of countries, which limits their comparability across countries. ${ }^{21}$ Moreover, Akseer and colleagues ${ }^{25}$ used different indicators to measure the empowerment of women in Islamic countries. Several other group-level indicators, such as the Gender Gap Index,${ }^{26}$ the Gender Development Index and the Gender Inequality Index, ${ }^{27}$ have been proposed at the country level. Subnational analysis or subgroup comparisons are not possible with these indicators. To better capture the multidimensional and complex construct of women's empowerment, Ewerling and colleagues developed the SWPER index based on individual-level DHS data from 34 African countries to enable comparability between countries and over time. ${ }^{21}$ SWPER includes three dimensions of women's empowerment that are indicative of assets and agency: social independence, decision-making and attitude to violence. ${ }^{21}$ Recently, SWPER Global has been developed and validated in 62 low-income and middle-income countries including Bangladesh. ${ }^{22}$ The SWPER index was constructed using a common set of questions from the DHS data and subsequently validated with the widely used Gender Development Index. The SWPER enables researchers to investigate the role of women's empowerment on maternal and child health intervention and outcomes. ${ }^{21}$
We have chosen the novel, multidimensional and validated SWPER index to examine the relationship between women's empowerment and maternal healthcare utilisation in Bangladesh. Bangladesh is a country with a deeply rooted patriarchy, characterised by women's limited access to economic resources, male guardianship and control over women's life choices, male control over social institutions, the confinement of women to household tasks and to the private sphere, lack of female access to higher education and employment, and a high prevalence of violence against women. ${ }^{28}$ We hypothesised that empowerment among Bangladeshi women will be associated with maternal healthcare utilisation and that higher empowerment will be linked to higher healthcare utilisation. Findings from this study will inform future efforts for promoting healthcare utilisation and will assist with improving maternal and child health in Bangladesh and other similar settings.

\section{METHODS \\ Data}

This cross-sectional study analysed secondary data from the most recent BDHS, 2017-2018. We obtained data from MEASURE DHS Archive (https://dhsprogram. com/data/dataset/Bangladesh_Standard-DHS_2017. cfm?flag $=0)$. A detailed description for obtaining the access and permission to analyse the DHS data is available (https://dhsprogramcom/data/Using-DataSets-for-Analysiscfm). The BDHS is a nationally representative survey conducted by the National Institute for Population Research and Training in collaboration with ICF International and Mitra and Associates. Details of the survey methodology have been described elsewhere. ${ }^{4}$ BDHS uses a nationwide household-based sample created via a stratified, two-stage cluster sampling procedure. In the first stage, census enumeration areas were selected using the probability proportional to size sampling technique. In the second stage, households were selected from the complete listing of households within a selected enumeration area via systematic random sampling. In these households, a total of 20376 ever-married women aged 15-49 years were identified, and 20127 were interviewed (response rate 99\%). This current analysis is restricted to 4767 currently married women who had a live birth in the 3 years preceding the survey. A flowchart of the sample selection for the present study is shown in figure 1 . The BDHS 2017-2018 was approved by the International Institutional Review Boards at ICF and the Bangladesh Medical Research Council. The BDHS conformed to international ethical standards of confidentiality, anonymity and informed consent. ${ }^{4}$ This study did not require further ethics approval because it used retrospective publicly available data.

\section{Outcomes}

Utilisation of MHS was our primary outcome of interest. MHS utilisation includes (1) receiving at least one ANC 


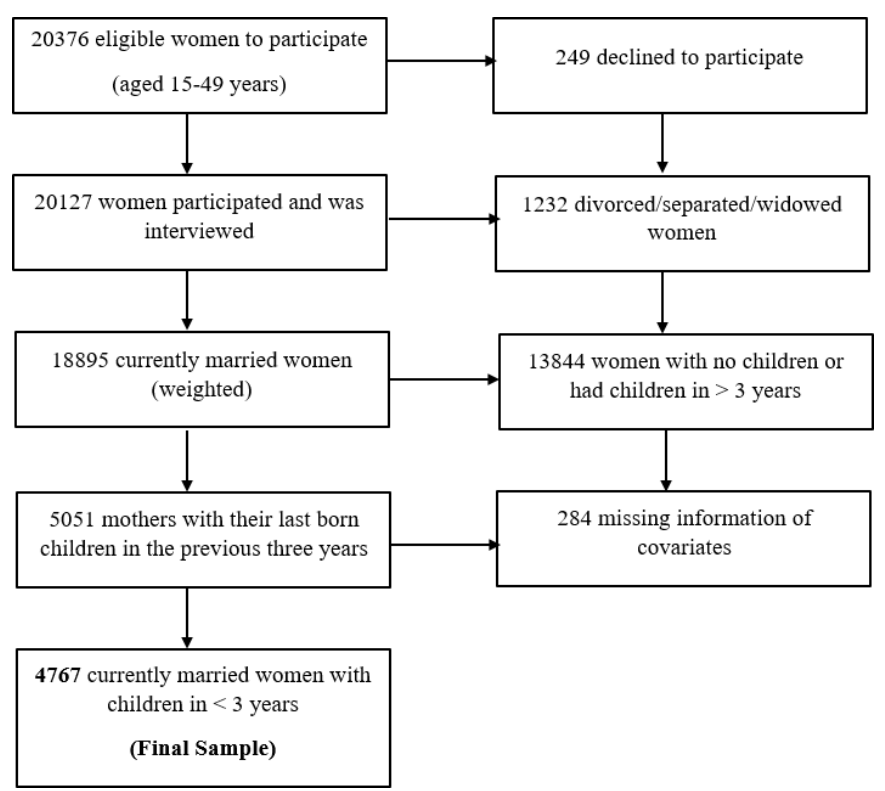

Figure 1 A flowchart for sample selection.

from skilled providers (ANC1), (2) receiving four or more ANC ( $\geq 4$ ANC), (3) delivery assisted by a skilled birth attendant (SBA) and (4) receiving postnatal care (PNC) for mother from skilled providers within 2 days of delivery. Skilled or medically trained providers for ANC and PNC include a qualified doctor, nurse, midwife, paramedic, family welfare visitor, community SBA, and sub-assistant community medical officer. However, SBA excludes subassistant community medical officers. ${ }^{4}$ Responses for each of these outcomes were dichotomised.

\section{Exposure}

Different aspects of women's empowerment as measured by the SWPER index were the main exposure of this study. The SWPER encompasses three domains of women's empowerment: attitude to violence, social independence and decision-making. Attitude to violence is dominated by questions related to women's opinions about whether wife beating was justified in various scenarios. Social independence includes items related to education, frequency of reading newspapers or magazines, and age at birth of first child and at first cohabitation. Age and education differences between the woman and her husband also appear in this domain, however, with lower loadings. Decision-making, the third domain, encompasses questions about participation in certain household decisions. In total, 14 items were extracted from the DHS, and principal component analysis (PCA) factor loadings were used to construct the index. ${ }^{21}{ }^{22}$ The 14 weighted items that most represented each domain, as well as how they were coded for the analyses, are described in online supplemental table 1 . This study used the recently developed and standardised Global SWPER. ${ }^{22}$ We used the standard cut-off points suggested by the authors, which roughly divide global scores into terciles. ${ }^{22}$ Applying these suggested cut-off points, each SWPER domain was divided into categories of low, medium or high levels of empowerment.

\section{Covariates}

The following sociodemographic characteristics were controlled for maternal age (15-24, 25-34 and 35-49 years old), current work status (working, not working), birth order (1, 2-3, 4-5, and 6 and more), pregnancy intention (wanted, unwanted), place of residence (urban, rural) and administrative region (Barisal, Chittagong, Dhaka, Khulna, Mymensingh, Rajshahi and Sylhet). Household socioeconomic status (SES) was derived from the household wealth index reported in the BDHS. SES was calculated using PCA of the assets owned by households; the detailed analytical procedures have been described previously. ${ }^{29}$ Households were ranked based on wealth scores and divided into quintiles with $1=$ poorest and $5=$ wealthiest .

\section{Statistical analysis}

Descriptive statistics were calculated for the sample, and $\chi^{2}$ tests were used to investigate the bivariate relationships between women's empowerment status as well as other sociodemographic characteristics and maternal healthcare utilisation. The magnitude of the associations between different domains of women's empowerment and maternal healthcare utilisation was assessed by estimating adjusted ORs (AOR) with 95\% CIs via multivariable logistic regression. All covariates were entered simultaneously into the multiple regression model. Finally, to measure the inequality of women's utilisation of MHS according to empowerment status, we used two regression-based methods: (1) relative index of inequality (RII) and (2) slope index of inequality (SII). The SII was determined by linear regression and the RII was computed using a modified Poisson approach. ${ }^{30}$ These two inequality indices have been previously used for the measurement of risks for socioeconomic and educational inequalities in different public health issues. ${ }^{31}{ }^{32} \mathrm{~A}$ positive SII indicates that maternal healthcare utilisation increases with the step-by-step increase of empowerment status (low to high), while a negative value means that utilisation decreases with increasing empowerment status; 0 indicates no association. Values of RII $>1$ indicate that women with high levels of empowerment are more likely to use MHS compared with the women with low empowerment. We set $\alpha=0.05$ for significance of all statistical tests. To control the effect of the complex survey (DHS) design, analyses were performed using Stata software's 'SVY' command. Variance inflation factors (VIFs) were used to evaluate the possible collinearity among the study variables; in all cases, the VIF values were close to unity, indicating that multicollinearity was not an issue. Stata V.14.2 was used for all statistical analyses.

\section{Patient and Public Involvement}

Patients or the public were not involved in the development of research questions, design of the study, 


\begin{tabular}{|c|c|c|}
\hline Characteristics & Frequency, n & $\%$ \\
\hline \multicolumn{3}{|c|}{ Respondent's age (in years) } \\
\hline $15-24$ & 2609 & 54.7 \\
\hline $25-34$ & 1889 & 39.6 \\
\hline $35-49$ & 269 & 5.7 \\
\hline \multicolumn{3}{|l|}{ Working status } \\
\hline Not working & 2986 & 62.7 \\
\hline Currently working & 1780 & 37.4 \\
\hline \multicolumn{3}{|l|}{ Birth order } \\
\hline 1 & 1771 & 37.2 \\
\hline $2-3$ & 2397 & 50.3 \\
\hline $4-5$ & 500 & 10.5 \\
\hline 6 and more & 99 & 2.0 \\
\hline \multicolumn{3}{|l|}{ Pregnancy intention } \\
\hline Intended & 3761 & 78.9 \\
\hline Unintended & 1006 & 21.1 \\
\hline \multicolumn{3}{|l|}{ Wealth index } \\
\hline Poorest & 1009 & 21.2 \\
\hline Poorer & 992 & 20.8 \\
\hline Middle & 909 & 19.1 \\
\hline Richer & 956 & 20.0 \\
\hline Richest & 902 & 18.9 \\
\hline \multicolumn{3}{|l|}{ Place of residence } \\
\hline Urban & 1253 & 26.3 \\
\hline Rural & 3514 & 73.7 \\
\hline \multicolumn{3}{|l|}{ Region } \\
\hline Barisal & 274 & 5.7 \\
\hline Chittagong & 1021 & 21.4 \\
\hline Dhaka & 1203 & 25.2 \\
\hline Khulna & 440 & 9.2 \\
\hline Mymensingh & 408 & 8.6 \\
\hline Rajshahi & 556 & 11.7 \\
\hline Rangpur & 508 & 10.7 \\
\hline Sylhet & 357 & 7.5 \\
\hline
\end{tabular}

recruitment and conduct of the study, or dissemination of the study results.

\section{RESULTS}

\section{Participant characteristics and trend of MHS utilisation}

Table 1 shows that $54.7 \%$ of respondents were aged $15-24$ years and the majority $(62.7 \%)$ were not working at the time of the survey. Approximately one-fifth of the most recent pregnancies $(21.1 \%)$ were reported as unintended. Results indicated that $26.3 \%$ of the women were from urban areas. Different aspects of women's empowerment assessed using the SWPER index are shown in figure 2. More than half the women reported that they possessed high empowerment relative to attitude to violence $(60 \%)$ and decision-making $(54.2 \%)$, while only



Figure 2 Percentages of different aspects of women's empowerment (survey-based Women's emPowERment (SWPER) index) by place of residence.

$27.3 \%$ of women reported high empowerment related to social independence. The proportion of high empowerment in all domains was higher among urban women than their rural counterparts (figure 2). Figure 3 displays the percentage of currently married women aged $15-49$ years who used MHS as well as the trend of using these services. In the last 15 years (2004-2018), maternal healthcare utilisation among women in Bangladesh increased significantly. ANC1 increased from $49.1 \%$ in 2004 to $83.5 \%$ in $2018, \geq 4$ ANC increased from $16.1 \%$ to $46.3 \%$, SBA increased from $14.2 \%$ to $51.9 \%$, and PNC increased from $14.9 \%$ to $50.9 \%$.

\section{Patterns of MHS utilisation by sociodemographics and domains of women's empowerment}

The proportion of MHS utilisation was significantly higher among women with high empowerment regarding attitude to violence compared with women who reported low empowerment: ANC1 (87.4\% vs 79.2\%; $<<0.001), \geq 4$ ANC (52.8\% vs $37.2 \%$; $\mathrm{p}<0.001)$, SBA $(59.8 \%$ vs $44.4 \%$; $\mathrm{p}<0.001)$ and PNC (58.2\% vs $43.9 \%$; $\mathrm{p}<0.001)$ (table 2). Higher utilisation of all MHS was also observed among women with high empowerment in both social independence and decision-making domains relative to their counterparts with low empowerment in these domains.

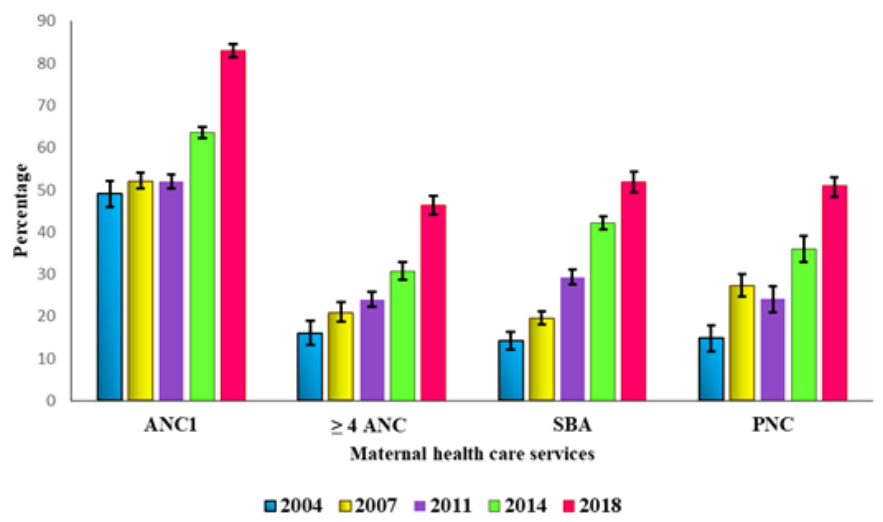

Figure 3 Trend of maternal healthcare utilisation among mothers in Bangladesh 2004-2018. ANC, antenatal care; ANC1, one antenatal care; PNC, postnatal care; SBA, skilled birth attendant. 
Table 2 Bivariate analysis of the relationship between women's empowerment and other sociodemographic variables and utilisation of maternal healthcare services among women, BDHS, $2018(\mathrm{~N}=4767)$

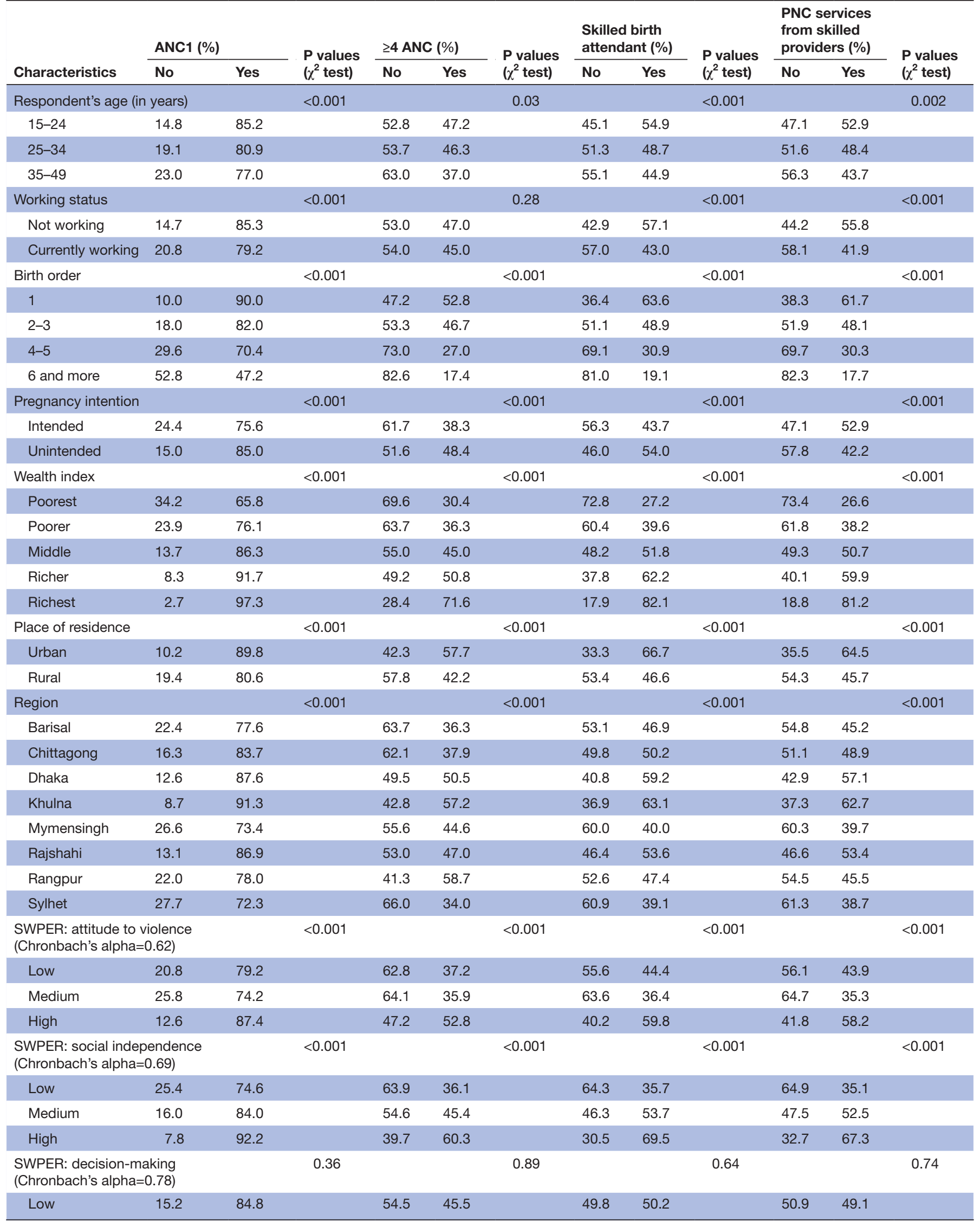


Table 2 Continued

\begin{tabular}{|c|c|c|c|c|c|c|c|c|c|c|c|c|}
\hline \multirow[b]{2}{*}{ Characteristics } & \multicolumn{2}{|c|}{ ANC1 (\%) } & \multirow{2}{*}{$\begin{array}{l}\text { P values } \\
\left.\text { ( } \chi^{2} \text { test }\right)\end{array}$} & \multicolumn{2}{|c|}{$\geq 4$ ANC (\%) } & \multirow{2}{*}{$\begin{array}{l}\text { P values } \\
\left(\chi^{2} \text { test }\right)\end{array}$} & \multicolumn{2}{|c|}{$\begin{array}{l}\text { Skilled birth } \\
\text { attendant (\%) }\end{array}$} & \multirow{2}{*}{$\begin{array}{l}\text { P values } \\
\left(\chi^{2} \text { test }\right)\end{array}$} & \multicolumn{2}{|c|}{$\begin{array}{l}\text { PNC services } \\
\text { from skilled } \\
\text { providers }(\%)\end{array}$} & \multirow{2}{*}{$\begin{array}{l}\text { P values } \\
\left.\text { ( } \chi^{2} \text { test }\right)\end{array}$} \\
\hline & No & Yes & & No & Yes & & No & Yes & & No & Yes & \\
\hline Medium & 16.7 & 83.3 & & 53.2 & 46.8 & & 47.3 & 52.7 & & 49.0 & 51.0 & \\
\hline High & 17.7 & 82.3 & & 53.7 & 46.3 & & 48.1 & 51.9 & & 49.2 & 50.8 & \\
\hline Total & 17.0 & 83.0 & & 53.7 & 46.3 & & 48.1 & 51.9 & & 49.1 & 50.9 & \\
\hline
\end{tabular}

$\geq 4$ ANC, four and more ANC visits; ANC1, at least one ANC visit from skilled providers; BDHS, Bangladesh Demographic and Health Survey; PNC, postnatal checkup of mother within 2 days after delivery from skilled providers; SBA, delivery by a skilled attendant; SWPER, survey-based Women's emPowERment.

Besides these empowerment domains, maternal age, working status, wealth index, pregnancy intention, place of residence, order of birth and region were significantly associated with maternal utilisations of healthcare services (table 2).

\section{Association between MHS utilisation and domains of women's empowerment}

Women with high empowerment in the attitude to violence domain were more likely than women with low empowerment to $\geq 4$ ANC (AOR 1.33; 95\% CI 1.10 to 1.60) (table 3). Additionally, compared with women with the least social independence empowerment, highly empowered women were more likely to use ANC1 (AOR $1.85 ; 95 \%$ CI 1.40 to 2.45 ), $\geq 4$ ANC (AOR $1.55 ; 95 \%$ CI 1.27 to 1.90 ), SBA (AOR 2.12; 95\% CI 1.71 to 2.62 ) and PNC (AOR 1.95; 95\% CI 1.56 to 2.44). Finally, highly empowered women relative to decision-making were 1.49 times (95\% CI 1.21 to 1.83 ) and 1.47 times (95\% CI 1.19 to 1.81 ) significantly more likely to use SBA and PNC services, respectively, than women with low empowerment in decision-making (table 3).

\section{Inequality in MHS utilisation by empowerment domain}

Moving from low empowerment to high empowerment in attitude to violence was associated with a $2 \%, 14 \%$, $13 \%$ and $12 \%$ increase in using ANC1, $\geq 4$ ANC, SBA and PNC, respectively (table 4). The corresponding SII for all indicators (ANC1, $\geq 4$ ANC, SBA and PNC) showed that a one-unit change from low to high empowerment in attitude to violence was associated with 5.81, 6.22, 6.27 and 5.77 per cent point increases for utilisation of all MHS indicators, respectively (table 4). Similarly, moving from low to high empowerment related to social independence was positively associated with a 3\%, 18\%, 19\% and $18 \%$ increase in using ANC1, $\geq 4$ ANC, SBA and PNC, respectively. However, in the decision-making domain, moving from low to high empowerment was significantly associated with SBA (5\%) and PNC (4\%) (table 4).

\begin{tabular}{|c|c|c|c|c|c|c|c|c|}
\hline $\begin{array}{l}\text { Women } \\
\text { Empowerment } \\
\text { Index (SWPER) }\end{array}$ & $\begin{array}{l}\text { ANC1 } \\
\text { AOR* }^{\star}(95 \% \mathrm{Cl})\end{array}$ & $P$ value & $\begin{array}{l}\geq 4 \text { ANC } \\
\text { AOR }^{\star}(95 \% \mathrm{Cl})\end{array}$ & $P$ value & $\begin{array}{l}\text { SBA } \\
\text { AOR }^{\star}(95 \% \mathrm{Cl})\end{array}$ & $P$ value & $\begin{array}{l}\text { PNC } \\
\text { AOR }^{\star}(95 \% \mathrm{Cl})\end{array}$ & $P$ value \\
\hline \multicolumn{9}{|c|}{ Attitude to violence } \\
\hline Low & 1 (ref) & & 1 (ref) & & 1 (ref) & & 1 (ref) & \\
\hline Medium & $0.91(0.70$ to 1.18$)$ & 0.47 & $1.06(0.85$ to 1.31$)$ & 0.60 & 0.79 (0.63 to 0.99$)$ & 0.04 & 0.76 (0.61 to 0.95$)$ & 0.02 \\
\hline High & $1.13(0.88$ to 1.45$)$ & 0.34 & 1.33 (1.10 to 1.60$)$ & 0.004 & 1.18 (0.96 to 1.46$)$ & 0.11 & 1.16 (0.94 to 1.43$)$ & 0.17 \\
\hline \multicolumn{9}{|c|}{ Social independence } \\
\hline Low & 1 (ref) & & 1 (ref) & & 1 (ref) & & 1 (ref) & \\
\hline Medium & $1.23(1.01$ to 1.50$)$ & 0.04 & 1.16 (0.98 to 1.37$)$ & 0.08 & $1.56(1.32$ to 1.84$)$ & $<0.001$ & 1.50 (1.26 to 1.78$)$ & $<0.001$ \\
\hline High & 1.85 (1.40 to 2.45$)$ & $<0.001$ & 1.55 (1.27 to 1.90$)$ & $<0.001$ & 2.12 (1.71 to 2.62$)$ & $<0.001$ & 1.95 (1.56 to 2.44 ) & $<0.001$ \\
\hline \multicolumn{9}{|l|}{ Decision-making } \\
\hline Low & 1 (ref) & & 1 (ref) & & 1 (ref) & & 1 (ref) & \\
\hline Medium & 1.01 (0.75 to 1.38$)$ & 0.92 & 1.11 (0.88 to 1.40$)$ & 0.40 & 1.26 (1.01 to 1.59$)$ & 0.04 & 1.22 (0.97 to 1.53$)$ & 0.09 \\
\hline High & 1.10 (0.82 to 1.48$)$ & 0.65 & 1.17 (0.95 to 1.43$)$ & 0.14 & 1.49 (1.21 to 1.83$)$ & $<0.001$ & 1.47 (1.19 to 1.81$)$ & $<0.001$ \\
\hline
\end{tabular}

*Adjusted by respondent's age, working status, birth order, pregnancy intention, wealth index, place of residence and region.

ANC1, at least one ANC visit from skilled providers; $\geq 4$ ANC4, four and more ANC visits; AOR, adjusted OR; BDHS, Bangladesh Demographic and Health Survey;

PNC, postnatal check-up of mother within 2 days after delivery; SBA, delivery by skilled attendant; SWPER, survey-based Women's emPowERment. 
To our knowledge, this study is the first to use nationally representative data to investigate the relationship between different aspects of women's empowerment as measured by the SWPER index and maternal healthcare utilisation among reproductive aged (15-49 years) women in Bangladesh. This study found that Bangladesh has experienced a significant increase in utilisation of MHS over the last 15 years. Our study also showed that women who had lower levels of empowerment, especially in the social independence domain, were least likely to use antenatal care, delivery services and postnatal care compared with their counterparts at higher empowerment levels. Although this study used a newly developed index (SWPER) to measure women's empowerment status, the relationships between women's empowerment and utilisation of maternal healthcare found in this study are consistent with previous studies around the world which used different measures of women's empowerment. ${ }^{10-19}$ Therefore, the findings of this study reaffirmed the association between women's empowerment and maternal healthcare utilisation in a patriarchal society such as Bangladesh.

Imbalances in gender power are high in Bangladesh, and thus women are generally dependent on their husband or male partner. However, this scenario is changing slowly due to government initiatives over the last few decades to empower women. Empowering women may produce normative changes in gender relations and women's role within the family and the community. ${ }^{33}$ These changes bring with them attendant changes in men's behaviour vis-à-vis women, including decisions about using maternal healthcare. With higher levels of empowerment, women may be able to improve their status at the domestic level, maintain their health and seek necessary health-related resources. ${ }^{34}$ Our investigation of the association between different dimensions of the SWPER index and maternal healthcare utilisation found that the social independence domain showed positive and significant associations with all the indicators of MHS, while attitude to violence was associated with $\geq 4$ ANC and decision-making with SBA and PNC. Moreover, inequality in using MHS between women with high and low empowerment statuses was present in all three domains.

\section{Attitude to violence domain and utilisation of MHS}

The attitude to violence domain showed a positive association with $\geq 4 \mathrm{ANC}$ in this study. Due to regional diversity and male-dominant norms, the acceptability of genderbased violence is widespread and inversely related to health-seeking behaviour in many African and South Asian countries. ${ }^{35}$ However, consistent with previous studies both from Bangladesh and elsewhere, ${ }^{193637}$ we found that women who did not justify violence were more likely to use MHS than women who justified violence. For example, in a recent Bangladeshi study, Tareque and colleagues $^{19}$ reported that compared with women who 
accept wife beating in at least one circumstance, women who did not accept it in any circumstance were 1.89 times and 1.93 times significantly more likely to use one or more antenatal care and delivery services from professionals, respectively. This higher utilisation of MHS by women with a high level of empowerment in attitude to violence could be due to their higher sense of entitlement. Not justifying wife beating for any reason is indicative of a greater sense of entitlement, self-esteem and status which positively reflect a woman's sense of empowerment and ability to claim their rights. ${ }^{48}$ The association found in this study, along with similar findings from other studies, ${ }^{14} 18193637$ indicates that women's attitude towards violence needs to be considered a vital sociocultural factor for utilisation of MHS during pregnancy.

\section{Social independence domain and utilisation of MHS}

The social independence domain, which comprises the frequency of reading print media, women's education, age at first birth, age at first cohabitation, and age and education differences between the woman and her husband, had greater magnitude and association strength relative to other domains in predicting the utilisation of MHS. This finding corroborates the results of previous studies. ${ }^{15213940}$ The social independence domain reflects a women's enlightenment and status, which may be related to knowledge of the importance of using MHS during and after pregnancy. Supporting this statement, a recent study in Benin documented a higher utilisation of antenatal care services among women with medium or high levels of enlightenment relative to women with low levels. ${ }^{41}$ Furthermore, all the variables included in the social independence domain have been individually reported to be protective factors for maternal healthcare utilisation. ${ }^{131418} 4243$ For example, a multicountry study by Sohn $e a^{13}$ reported that media access was associated with higher maternal healthcare utilisation. The variety of items in this domain and their individual relationships with maternal healthcare utilisation could be a possible reason for the strength of social independence in predicting maternal healthcare utilisation.

\section{Decision-making domain and utilisation of MHS}

This study found a significant relationship between the decision-making domain of the SWPER index and utilisation of SBA and PNC services. Consistent with our findings, Aziz and colleagues ${ }^{15}$ reported that high autonomy in household decision-making was significantly associated with higher maternal healthcare-seeking behaviour. In addition, several other studies in developing countries suggested that women's decision-making power in the household was an important factor for the appropriate utilisation of MHS. ${ }^{15} 18204044$ Women with higher decision-making power have greater influence within their households and in society and are more likely to use healthcare services for themselves and their children. ${ }^{45}$ Additionally, higher decision-making power and control over household resources allows a woman to more capably communicate with family members and to demand care for herself when needed. ${ }^{46}$ However, in contrast to our findings, some other results indicated no association between women's decision-making power and maternal healthcare utilisation, ${ }^{14} 1648$ suggesting the need for further in-depth investigation.

\section{Strengths and limitations}

Our study has several strengths. While earlier studies used only one or two components of empowerment, we used the multidimensional SWPER index to assess the relationship between women's empowerment and utilisation of MHS in Bangladesh. Additionally, we used the most recent BDHS data, which is a nationally representative sample including both rural and urban areas with a large sample size that permits precise estimates. However, this study has limitations as well. First, the concept of women's empowerment is complex, including economic, sociocultural, familial, interpersonal, legal, political, societal and psychosocial dimensions. Although the SWPER index includes three domains of empowerment, fully capturing all dimensions of empowerment in a single index is difficult. Furthermore, an association found at an aggregated level does not necessarily represent an association at an individual level. In addition, some measures used in the SWPER index, such as attitude to violence, may be subject to social desirability bias and thus under-reported. Second, utilisation of MHS was self-reported and was not confirmed by medical records, and so may be subject to recall bias. Additionally, the data used in this study were from currently married women who had a live birth within the 3 years preceding the survey, so the findings should not be generalised to women of all ages. Third, this study was based on secondary data, which limits the analysis to the variable that are available. Therefore, our adjusted analyses did not include all potential factors that could influence women's healthcare-seeking behaviour, such as cost of care, availability and accessibility of health facilities, equity in health service delivery, timing of ANC visits, or knowledge and attitudes towards modern healthcare services. Finally, this study is cross-sectional and therefore we cannot assert causality or specify the direction of the effects.

\section{Policy implications}

The study findings have important policy implications and far-reaching consequences for improving maternal and child health in Bangladesh and other similar settings. Different domains of empowerment were found to be significantly associated with maternal healthcare-seeking behaviours. The finding implies that to achieve the UN's Sustainable Development Goals 3.1 (reducing the global MMR to less than 70 per 100000 live births by 2030) and 3.2 (reducing neonatal mortality to at least as low as 12 per 1000 live births and under-5 mortality to at least as low as 25 per 1000 live births), policies with the potential to increase women's empowerment should be articulated and implemented. Future efforts should strive to promote 
women's empowerment, with a particular concentration on the social independence domain. Since more than half the women in our sample visited health facilities at least once, interventions to promote empowerment could be implemented at healthcare facilities with appropriate staff training. Although more research is warranted to identify the most effective strategies to empower women in Bangladesh, the health system could be an entry point for addressing gender imbalances. Lastly, since women's healthcare utilisation largely depends on their husbands, efforts to address these issues should include men to educate them about the importance of health services to promote and maintain the family's health. Therefore, we suggest that interventions must target men and women together to promote health education.

\section{CONCLUSION}

This study used the globally developed and validated SWPER index to evaluate women's empowerment, and thus provides a greater understanding of the wide ranging and important relationship between women's empowerment and utilisation of MHS in Bangladesh. This study found that women's empowerment was positively associated with several key indicators of MHS and therefore suggests that policies and actions to promote women's empowerment could be effective in higher utilisation of MHS, consequently improving maternal and child health outcomes. However, further research is needed to identify wide-ranging confounding factors that may influence the cause-and-effect relationship between specific aspects of women's empowerment that effectively influence the utilisation of MHS.

Acknowledgements We are thankful to the MEASURE DHS for serving us with the latest dataset. A special thanks and heartfelt gratitude goes to Thomas W. Pullum, $\mathrm{PhD}$ (Director of Research, The Demographic and Health Surveys Program, ICF) for his unconditional help and consultation during conceptualising the research.

Contributors AIA and MMR conceptualised and designed the study. AIA curated, analysed and interpreted the data, and wrote the first draft. MMR supervised the study. MMR and BG critically review the manuscript with important intellectual inputs. All authors commented on and approved the final manuscript.

Funding The authors have not declared a specific grant for this research from any funding agency in the public, commercial or not-for-profit sectors.

Competing interests None declared.

Patient consent for publication Not required.

Ethics approval The BDHS 2017-18 was approved by International Institutional Review Boards at ICF (ICF IRB FWA00000845) and the Bangladesh Medical Research Council (BMRC) (BMRC/NREC/2016-2019/324). The BDHS conformed to international ethical standards of confidentiality, anonymity and informed consent. This study did not require further ethics approval because it used retrospective publicly available data.

Provenance and peer review Not commissioned; externally peer reviewed.

Data availability statement Data are available in a public, open access repository. Data are available on the website (https://dhsprogram.com/data/AccessInstructions.cfm).

Supplemental material This content has been supplied by the author(s). It has not been vetted by BMJ Publishing Group Limited (BMJ) and may not have been peer-reviewed. Any opinions or recommendations discussed are solely those of the author(s) and are not endorsed by BMJ. BMJ disclaims all liability and responsibility arising from any reliance placed on the content. Where the content includes any translated material, BMJ does not warrant the accuracy and reliability of the translations (including but not limited to local regulations, clinical guidelines, terminology, drug names and drug dosages), and is not responsible for any error and/or omissions arising from translation and adaptation or otherwise.

Open access This is an open access article distributed in accordance with the Creative Commons Attribution Non Commercial (CC BY-NC 4.0) license, which permits others to distribute, remix, adapt, build upon this work non-commercially, and license their derivative works on different terms, provided the original work is properly cited, appropriate credit is given, any changes made indicated, and the use is non-commercial. See: http://creativecommons.org/licenses/by-nc/4.0/.

\section{ORCID iDs}

Asibul Islam Anik http://orcid.org/0000-0002-6885-2256

Bishwajit Ghose http://orcid.org/0000-0003-4461-3821

Md. Mosfequr Rahman http://orcid.org/0000-0002-2402-5814

\section{REFERENCES}

1 World Health Organization. Trends in maternal mortality 2000 to 2017: estimates by WHO, UNICEF, UNFPA. World Bank Group and the United Nations Population Division, 2019.

2 World Bank Group, the United Nations Population Division. Trends in maternal mortality: 1990 to 2015: estimates by WHO, UNICEF, UNFPA. Geneva: WHO, 2015.

3 Shahabuddin ASM, Delvaux T, Abouchadi S, et al. Utilization of maternal health services among adolescent women in Bangladesh: a scoping review of the literature. Trop Med Int Health 2015;20:822-9.

4 National Institute of Population Research and Training (NIPORT), ICF. Bangladesh demographic and health survey 2017-18. Dhaka, Bangladesh, and Rockville, Maryland, USA: NIPORT and ICF, 2020.

5 World Health Organization. Standards for improving quality of maternal and newborn care in health facilities, 2016. https://www. who.int/docs/default-source/mca-documents/advisory-groups/ quality-of-care/standards-for-improving-quality-of-maternal-andnewborn-care-in-health-facilities.pdf

6 Akter S, Rich JL, Davies K, et al. Access to maternal healthcare services among indigenous women in the Chittagong Hill tracts, Bangladesh: a cross-sectional study. BMJ Open 2019;9:e033224.

7 Berri KM, Adaba YK, Tarefasa TG, et al. Maternal health service utilization from urban health extension professionals and associated factors among women who gave birth in the last one year in Ambo town, Oromia regional state, Ethiopia, 2018. BMC Public Health 2020;20:499.

8 Kruk ME, Galea S, Prescott M, et al. Health care financing and utilization of maternal health services in developing countries. Health Policy Plan 2007;22:303-10.

9 Nguyen HTH, Hatt L, Islam M, et al. Encouraging maternal health service utilization: an evaluation of the Bangladesh voucher program. Soc Sci Med 2012;74:989-96.

10 Ahmed S, Creanga AA, Gillespie DG, et al. Economic status, education and empowerment: implications for maternal health service utilization in developing countries. PLoS One 2010;5:e11190.

11 Jennings L, Na M, Cherewick M, et al. Women's empowerment and male involvement in antenatal care: analyses of demographic and health surveys (DHS) in selected African countries. BMC Pregnancy Childbirth 2014;14:297.

12 Shimamoto K, Gipson JD. The relationship of women's status and empowerment with skilled birth attendant use in Senegal and Tanzania. BMC Pregnancy Childbirth 2015;15:154.

13 Sohn M, Jung M. Effects of Empowerment and media use by women of childbearing age on maternal health care utilization in developing countries of Southeast Asia. Int J Health Serv 2020;50:32-43.

14 Mondal D, Karmakar S, Banerjee A. Women's autonomy and utilization of maternal healthcare in India: evidence from a recent national survey. PLoS One 2020;15:e0243553.

15 Aziz S, Kumar Mitra D, Omar Rahman M, Kumar MD, Omar RM. Identifying factors associated with low maternal health care seeking behavior in Bangladesh: does women's autonomy matter? Women's Reproductive Health 2017;4:115-25.

16 Ghose B, Feng D, Tang S, et al. Women's decision-making autonomy and utilisation of maternal healthcare services: results from the Bangladesh demographic and health survey. BMJ Open 2017;7:e017142.

17 Haider MR, Qureshi ZP, Khan MM. Effects of women's autonomy on maternal healthcare utilization in Bangladesh: evidence from a national survey. Sex Reprod Healthc 2017;14:40-7. 
18 Haque SE, Rahman M, Mostofa MG, et al. Reproductive health care utilization among young mothers in Bangladesh: does autonomy matter? Womens Health Issues 2012;22:e171-80.

19 Tareque MI, Alam MS, Peet ED, et al. Justification of wife beating and utilization of antenatal and delivery care in Bangladesh. $J$ Interpers Violence 2020;0886260519898444:088626051989844.

20 Piu ZA, Khanam M. Women empowerment and maternal healthcare service utilization: evidence from Bangladesh. Women Empowerment 2020;36 https://www.researchgate.net/publication/338526796 Women_Empowerment_and_Maternal_Healthcare_Service_ Utilization_Evidence_from_Bangladesh

21 Ewerling F, Lynch JW, Victora CG, et al. The SWPER index for women's empowerment in Africa: development and validation of an index based on survey data. Lancet Glob Health 2017:5:e916-23.

22 Ewerling F, Raj A, Victora CG, et al. SWPER global: a survey-based women's empowerment index expanded from Africa to all low- and middle-income countries. J Glob Health 2020;10:020434.

23 Bishop D, Bowman K. Still learning: a critical reflection on three years of measuring women's empowerment in Oxfam. Gender \& Development 2014;22:253-69.

24 Do M, Kurimoto N. Women's empowerment and choice of contraceptive methods in selected African countries. Int Perspect Sex Reprod Health 2012;38:023-33.

25 Akseer N, Kamali M, Bakhache N, et al. Status and drivers of maternal, newborn, child and adolescent health in the Islamic world: a comparative analysis. Lancet 2018;391:1493-512.

26 World Economic Forum. The global gender gap report. Geneva: WEF 2014.

27 Human development report 2015: work for human development2015United Nations Development Programme

28 Karim R, Lindberg L, Wamala S, et al. Men's perceptions of women's participation in development initiatives in rural Bangladesh. Am J Mens Health 2018;12:398-410.

29 Rutstein SO, Johnson K. The DHS wealth index. DHS comparative reports No. 6. Calverton: ORC Macro, 2004.

30 Zou G. A modified poisson regression approach to prospective studies with binary data. Am J Epidemiol 2004;159:702-6.

31 Arsenault C, Jordan K, Lee D, et al. Equity in antenatal care quality: an analysis of 91 national household surveys. Lancet Glob Health 2018;6:e1186-95.

32 Moreno-Betancur M, Latouche A, Menvielle G, et al. Relative index of inequality and slope index of inequality: a structured regression framework for estimation. Epidemiology 2015;26:518-27.

33 Koenig MA, Ahmed S, Hossain MB, et al. Women's status and domestic violence in rural Bangladesh: individual- and communitylevel effects. Demography 2003;40:269-88.
34 Fikree FF, Pasha O. Role of gender in health disparity: the South Asian context. BMJ 2004;328:823-6.

35 Sripad P, Warren CE, Hindin MJ, et al. Assessing the role of women's autonomy and acceptability of intimate-partner violence in maternal health-care utilization in 63 low- and middle-income countries. Int J Epidemiol 2019;48:1580-92.

36 Adhikari R. Effect of Women's autonomy on maternal health service utilization in Nepal: a cross sectional study. BMC Womens Health 2016;16:26.

37 Ahuru RR. The influence of women empowerment on maternal and childcare use in Nigeria. Int J Healthc Manag 2021;14:690-9.

38 Yount KM, Halim N, Schuler SR, et al. A survey experiment of women's attitudes about intimate partner violence against women in rural Bangladesh. Demography 2013;50:333-57.

39 Akter SM, Roy SK, Thakur SK, et al. Effects of third trimester counseling on pregnancy weight gain, birthweight, and breastfeeding among urban poor women in Bangladesh. Food Nutr Bull 2012;33:194-201.

40 Mallick L, Zafar R, Juan C, et al. Trends and the relationship between maternal health and empowerment in Pakistan, 2012-2018. Rockville, Maryland, USA: ICF, 2020.

41 Ekholuenetale M, Nzoputam Cl, Barrow A, et al. Women's enlightenment and early antenatal care initiation are determining factors for the use of eight or more antenatal visits in Benin: further analysis of the Demographic and Health Survey. J Egypt Public Health Assoc 2020;95:1-12.

42 Godha D, Hotchkiss DR, Gage AJ. Association between child marriage and reproductive health outcomes and service utilization: a multi-country study from South Asia. J Adolesc Health 2013;52:552-8

43 Sinha KC. Male involvement and utilization of maternal health services in India. Int J Sci Res Pub/2014;4:1-13 http://www.ijsrp.org/ research-paper-1114/ijsrp-p3544.pdf

44 Chol C, Negin J, Agho KE, et al. Women's autonomy and utilisation of maternal healthcare services in 31 sub-Saharan African countries: results from the demographic and health surveys, 2010-2016. BMJ Open 2019;9:e023128.

45 Self S, Grabowski R. Female autonomy and health care in developing countries. Rev Develop Econ 2012;16:185-98.

46 Bhatia JC, Cleland J. Determinants of maternal care in a region of South India. Health Transit Rev 1995:127-42 https://openresearchrepository.anu.edu.au/bitstream/1885/41182/2/Bhati1_1.pdf

47 Maitra P. Parental bargaining, health inputs and child mortality in India. J Health Econ 2004;23:259-91.

48 Furuta M, Salway S. Women's position within the household as a determinant of maternal health care use in Nepal. Int Fam Plan Perspect 2006;32:017-27. 